\title{
Editorial
}

Notfall Rettungsmed 2019 $\cdot 22: 181$

https://doi.org/10.1007/s10049-019-0598-9

(c) Springer Medizin Verlag $\mathrm{GmbH}$, ein Teil von Springer Nature 2019

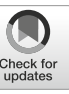

G. Rücker $r^{1}$ B. Dirks ${ }^{2} \cdot$ S. Wingen ${ }^{3} \cdot$ B. W. Böttiger ${ }^{3}$

${ }^{1}$ Klinik und Poliklinik für Anästhesiologie und Intensivtherapie, Rostocker Simulationsanlage und Notfallausbildungszentrum, Universitätsmedizin Rostock, Rostock, Deutschland

${ }^{2}$ Ulm, Deutschland

${ }^{3}$ Klinik für Anästhesiologie und Operative Intensivmedizin, Universitätsklinikum Köln (AöR), Köln, Deutschland

\section{KIDS SAVE LIVES - Modularer Lehrerausbildungskurs des Deutschen Rates für Wiederbelebung (GRC) für den Schulunterricht in Wiederbelebung}

Die Nähe des Begriffes zur „Laien-Wiederbelebung“ liegt da klar auf der Hand. So gesehen lief diese dem Trend lange voraus, ja involvierte sogar Kinder, nachdem man herausgefunden hatte, dass diese damit Menschenleben retten können. Die Kultusministerkonferenz trug dem 2014 Rechnung, indem sie sich klar zur Einführung von Wiederbelebungsunterricht in der Schule positionierte, um die Wiederbelebung durch Laienhelfer in Deutschland $\mathrm{zu}$ stärken. Das war die logische Konsequenz aus der jahrelangen Bemühung zur Steigerung der Überlebensrate nach einem plötzlichen Herzstillstand.

Es ist nun an der Zeit, dieses Projekt flächendeckend umzusetzen. Der Deutsche Rat für Wiederbelebung (German Resuscitation Council, GRC, www.grcorg.de) hat in einem Konsensuspapier unter Beteiligung der Hilfsorganisationen, Vertretern des Bundesministeriums für Gesundheit sowie Schulvertretern ein Curriculum für Lehrkräfte erarbeitet. Hierdurch sollen Lehrer ausgebildet werden, um selbst den Wiederbelebungsunterricht in den Schulen durchführen zu können. Kernbaustein ist ein 4-stündiger Kurs, der zunächst durch qualifizierte GRC-Kursinstruktoren bundesweit angeboten wird. Ein nachhaltiges Konzept ist geboren, das der Empfehlung der Kultusministerkonferenz entspricht und bei der langfristigen Etablierung des Wiederbelebungsunterrichtes in den Schulen unterstützt. Das Ganze fällt in eine Zeit, in der auch die Hilfsorganisationen die Wiederbelebungsausbildung aus ihrem klassischen Erste-Hilfe-Konzept für die Erwachsenenausbildung herausgelöst haben und diese jetzt als Zusatzbaustein anbieten. Die Wiederbelebungsausbildung ist damit bei Schülern und Erwachsenen sehr gut aufgestellt, und wir dürfen voller Hoffnung sein, dass sich mit diesen Maßnahmen ein großer Sprung nach vorne in der Laienwiederbelebungsbeteiligung erzielen lässt.

\section{Korrespondenzadresse}

Univ.-Prof. Dr. med. B. W. Böttiger

Klinik für Anästhesiologie und Operative Intensivmedizin, Universitätsklinikum Köln (AöR)

Kerpener Straße 62, 50937 Köln, Deutschland bernd.boettiger@uk-koeln.de

Interessenkonflikt. G. Rücker, B. Dirks, S. Wingen und B.W. Böttiger geben an, dass kein Interessenkonflikt besteht.
Aus Gründen der besseren Lesbarkeit wird in dieser Fachpublikation in der Regel das generische Maskulinum als geschlechtsneutrale Form verwendet. 